\begin{tabular}{lcl}
\hline INESEG \\
INTERNATIONAL \\
ENGINEERING, \\
SCIENCE AND \\
EDUCATION GROUP
\end{tabular} Middle East Journal of Science $_{\text {e-ISSN:2618-6136 }}$

Research Article

\title{
NEW EXACT SOLUTIONS FOR KLEIN-GORDON EQUATION
}

\author{
Faruk Düşünceli*1 (D) \\ ${ }^{1}$ Mardin Artuklu University Departmant of Economics \\ *Corresponding author; farukdusunceli@artuklu.edu.tr
}

\begin{abstract}
In this paper, we applied the improved Bernoulli sub-equation function method for the KleinGordon equation. Firstly, we reduced the equation to a nonlinear ordinary differential equation with the aid of wave transform. Then we obtained various new exact solutions via the method. These solutions can play an important role in engineering and physics. For some solutions, we drew two and threedimensional graphics to understand physical behaviors. We performed all the calculations and graphs in this article by Wolfram Mathematica.
\end{abstract}

Keywords: Klein-Gordon Equation, Improved Bernoulli sub-equation function method, Exact solutions.

Received: September 21, 2020

Accepted: November 16, 2020

\section{Introduction}

Nonlinear evolution equations (NLEEs) are widely used because it finds application in many nonlinear disciplines such as plasma physics, optical fibers, fluid mechanics, fluid dynamics[1-4] and so on. One of the best known of these equations is Klein-Gordon equation (KGE). KGE, the relative wave equation version of Schrödinger equation, is a relative field equation for scalar particles (spin-zero) [5].

Sassaman and Biswas[5,6] investigated the general form of the perturbed KGE,

$$
u_{t t}-u_{x x}+f(u)=\varepsilon\left(\alpha u+p u_{t}+q u_{x}+\beta u_{x t}+\gamma u_{t t}\right)
$$

where $\alpha, \beta, \gamma, p, q$ are constants and $\varepsilon$ is the perturbation parameter. Zhang [7] investigated this equation without local inductance and dissipation effect:

$$
u_{t t}-u_{x x}+f(u)=\varepsilon\left(\alpha u+\beta u_{x t}+\gamma u_{t t}\right)
$$

When $f(u)=a u-b u^{2}$ and $\varepsilon=0$, Eq.(1) degrades the KGE with quadratic nonlinearity:

$$
u_{t t}-u_{x x}+a u-b u^{2}=0
$$


When $f(u)=a u-b u^{3}$ and $\varepsilon=0$, Eq.(1) degrades the KGE with cubic nonlinearity:

$$
u_{t t}-u_{x x}+a u-b u^{3}=0
$$

Equation (4) has been solved by many different methods in the literature. Zhang [8] used the trigonometric function series method. Wazwaz[9] applied the tanh and sine-cosine methods. Hafez et al. [10] solved it with $\frac{G^{\prime}}{G}$ expansion method. Yindoula et al. [11] solved it with He-Laplace and the Decomposition Laplace-Adomian method. Lastly, Shahen et al. [12] used the $\exp (-\varphi(\varepsilon) \operatorname{expansion}$ method.

In this paper, we solved equation (4) by improved Bernoulli sub-equation function method (IBSEFM). The remainder of this paper is prepared as follows: In section 2, the method has been discussed. In section 3, we applied this method to the KGE with cubic nonlinearity. Lastly, conclusions are given in section 4 .

\section{Material and method}

Improved Bernoulli sub-equation function method [13-17] formed by modifying the Bernoulli sub-equation function method will be introduced in this section. The algorithm below should be applied sequentially.

1. Let's consider the following partial differential equation;

$$
P\left(u, u_{x}, u_{y}, \ldots, u_{x x}, u_{x y}, \ldots=0\right.
$$

and take the wave transformation;

$$
u(x, y, t, \ldots)=U(\eta), \eta=k x+l y+m t+\cdots
$$

where $k, l, m, \ldots$ are constants and will be determined later. Putting Equation (3) in Equation (2), we get the following nonlinear ordinary differential equation;

$$
N\left(U, U^{\prime}, U^{\prime \prime}, U^{\prime \prime \prime}, \cdots\right)=0
$$

2. Considering the trial equation in Equation (4), it can be written as follows;

$$
U(\eta)=\frac{\sum_{i=0}^{n} a_{i} F^{i}(\eta)}{\sum_{j=0}^{m} b_{j} F^{j}(\eta)}=\frac{a_{0}+a_{1} F(\eta)+a_{2} F^{2}(\eta)+\cdots+a_{n} F^{n}(\eta)}{b_{0}+b_{1} F(\eta)+b_{2} F^{2}(\eta)+\cdots+b_{m} F^{m}(\eta)}
$$


According to Bernoulli theory, we can consider the general form of the Bernoulli differential equation for $F^{\prime}$ as follows:

$$
F^{\prime}=w F+d F^{M}, w, d \neq 0, M \in R-\{0,1,2\}
$$

Where $F=F(\eta)$ is Bernoulli differential polynomial. By replacing the above relations with Equation (4), it gives the equations of polynomial $\varphi(F)$ as follows;

$$
\varphi(F)=\rho_{s} F^{s}+\cdots+\rho_{1} F+\rho_{0}=0
$$

According to the balance principle, we can determine the relationship between $n, m$ and $M$.

3. The coefficients of (F) must all be zero and give us an algebraic system of equations;

$$
\rho_{i}=0, i=0, \cdots, s
$$

Solving this system, we will determine the values of $a_{0}, \cdots, a_{n}$ and $b_{0}, \cdots, b_{m}$.

4. When we solve the nonlinear Bernoulli differential equation (6), we get the following two cases with respect to $w$ and $d$;

$$
\begin{gathered}
F(\eta)=\left[\frac{-d}{w}+\frac{E}{e^{w(1-M) \eta}}\right]^{\frac{1}{1-M}}, w \neq d \\
F(\eta)=\left[\frac{(E-1)+(E+1) \tanh \left(w(1-M) \frac{\eta}{2}\right)}{1-\tanh \left(w(1-M) \frac{\eta}{2}\right)}\right]^{\frac{1}{1-M}}, w=d, E \in R
\end{gathered}
$$

\section{Findings}

In this section, the application of the improved Bernoulli sub-equation function method to the Klein-Gordon Equation is discussed. Using the wave transformation on equation (1)

$$
\varnothing(x, t)=U(\eta), \eta=k x-l t
$$

Substituting equation (11) into equation (1), gives the following NODE:

$$
\left(l^{2}+\alpha k^{2}\right) U^{\prime \prime}+a U-b U^{3}=0
$$


Balancing equation (12) by considering the highest derivative $\left(U^{\prime \prime}\right)$ and the highest power $\left(U^{3}\right)$, we obtain

$$
n-m=M-1
$$

When $M=3, m=1$, gives $n=3$. Thus, the trial solution for equation (1) takes the following form:

$$
U(\eta)=\frac{a_{0}+a_{1} F(\eta)+a_{2} F^{2}(\eta)+a_{3} F^{3}(\eta)}{b_{0}+b_{1} F(\eta)}
$$

Where $F^{\prime}=w F+d F^{3}, w \neq 0, d \neq 0$. Substituting equation (13), its second derivative along with $F^{\prime}=w F+d F^{3}, w \neq 0, d \neq 0$ into equation (12), gives an $\mathrm{F}$ polynomial. Solving the system of the algebraic equations gives the values of the relevant parameter. Substituting the obtained values of the parameters into equation (13), gives the solutions to equation (1).

$w \neq d$, We can find the following coefficients:

\section{Case 1.}

$$
a_{1}=\frac{a_{0} b_{1}}{b_{0}} ; a_{2}=\frac{2 d a_{0}}{\sigma} ; a_{3}=\frac{2 d a_{0} b_{1}}{\sigma b_{0}} ; w=d ; c=2\left(l^{2}+k^{2} \alpha\right) \sigma^{2} ; b=-\frac{2\left(l^{2}+k^{2} \alpha\right) \sigma^{2} b_{0}^{2}}{a_{0}^{2}} ;
$$

\section{Case 2.}

$$
a_{1}=\frac{a_{0} b_{1}}{b_{0}} ; a_{2}=0 ; a_{3}=0 ; \alpha=-\frac{l^{2}}{k^{2}} ; w=3 d ; a=-\frac{b a_{0}^{2}}{b_{0}^{2}}
$$

\section{Case 3.}

$$
a_{1}=\frac{a_{0} b_{1}}{b_{0}} ; a_{2} \rightarrow 0 ; a_{3} \rightarrow 0 ; l=-i k \sqrt{\alpha} ; a=-\frac{b a_{0}^{2}}{b_{0}^{2}}
$$

Substituting equation (14) into equation (13), gives

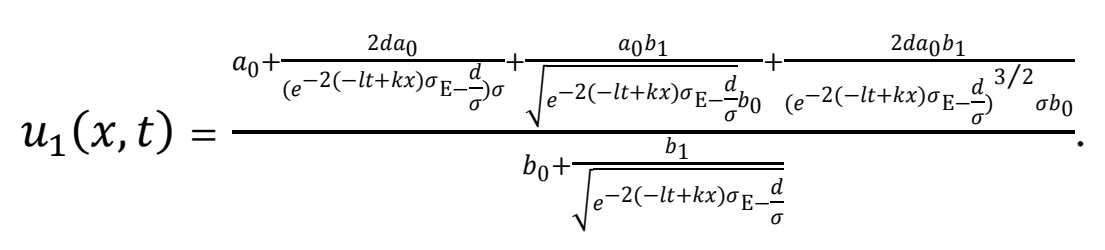

Substituting equation (15) into equation (13), gives 
Substituting equation (16) into equation (13), gives

$$
u_{2}(x, t)=\frac{a_{0}+\frac{a_{0} b_{1}}{\sqrt{e^{-2(-l t+k x) \sigma_{\mathrm{E}}-\frac{3 d}{\sigma}} b_{0}}}}{b_{0}+\frac{b_{1}}{\sqrt{e^{-2(-l t+k x) \sigma_{\mathrm{E}-}-\frac{3 d}{\sigma}}}}} .
$$

$$
u_{3}(x, t)=\frac{a_{0}+\frac{a_{0} b_{1}}{\sqrt{e^{-2(k x+i k t \sqrt{\alpha}) \sigma_{\mathrm{E}-}} \frac{w}{\sigma} b_{0}}}}{b_{0}+\frac{b_{1}}{\sqrt{e^{-2(k x+i k t \sqrt{\alpha}) \sigma_{\mathrm{E}-}-\frac{w}{\sigma}}}}} .
$$

We performed numerical simulations by selecting appropriate parameter values and drawing 2D and 3D graphs of the solutions obtained for equation (17).
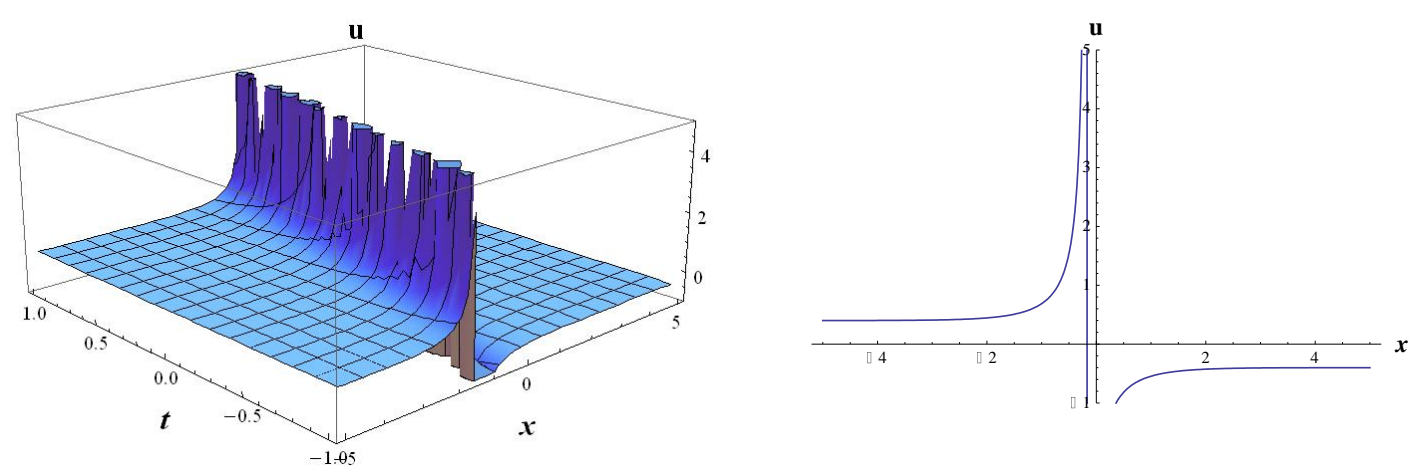

Figure 1. The 2D and 3D surfaces of the solution Eq.(17) for suitable values

\section{Conclusions}

IBSEF method has been employed to get new exact solutions for the Klein-Gordon Equation. When we compared the results we obtained with the previous ones, we saw that they were new solutions. The results that we obtained can be helpful in explaining the physical sense of the diverse nonlinear models that originated in the area of the nonlinear sciences. The method is a robust and efficient mathematical tool that can be used to manipulate some type of nonlinear mathematical model.. We recommend that this method can be applied to distinct nonlinear partial differential equations. We suggest applying this method to different nonlinear partial differential equations.

The compliance to the Research and Publication Ethics: This study was carried out in accordance with the rules of research and publication ethics.

\section{References}

[1] Akın, L ., "A Characterization of Approximation of Hardy Operators in VLS". Celal Bayar University Journal of Science, 14 (3), 333-336,2018. DOI: 10.18466/cbayarfbe.449954. 
[2] Mamedov, F, Zeren, Y, Akın, L, "Compactification of weighted Hardy operator in variable exponent Lebesgue spaces", Asian Journal of Mathematics and Computer Science, 17:1, 38$47,2017$.

[3] Akın, L. "On Some Properties of Integral-Type Operator in Weighted Herz Spaces with Variable Exponent Lebesgue Spaces" Universal Journal of Mathematics and Applications, 2 (3), 148151,2019. DOI: $10.32323 /$ ujma.522420

[4] Cruz-Uribe, D., Diening, L. and Hasto, P., "The maximal operator on weighted variable Lebesgue spaces”, Fract. Calc. Appl. Anal. 14(3), 361-374,2011.

[5] Biswas, A., Zony, C. and Zerrad, E., "Soliton perturbation theory for the quadratic nonlinear Klein-Gordon equation" Applied Mathematics and Computation, 203, 153, 2008.

[6] Sassaman, R., Biswas, A, "Soliton perturbation theory for phi-four model and nonlinear KleinGordon equations", Commun Nonlinear Sci Numer Simulat, 14, 3239-3249, 2009.

[7] Zhang, Z., "Exact traveling wave solutions of the perturbed Klein-Gordon equation with quadratic nonlinearity in (1+1)-dimension, Part I: Without local inductance and dissipation effect", Turkish Journal of Physics, 37, 259-267,2013.

[8] Zhang, Z., "New Exact Traveling Wave Solutions for the Nonlinear Klein-Gordon Equation", Turkish Journal of Physics, 32, 235-240,2008.

[9] Wazwaz, A. M. "The tanh and sine-cosine methods for compact and noncompact solutions of the nonlinear Klein-Gordon equation”, Applied Mathematics and Computation, 167(2), 1179-1195, 2005 .

[10] Hafez, M. G., Nur Alam, M. and Akbar, M.A. "Exact traveling wave solutions to the KleinGordon equation using the novel (G0/G)-expansion method", Results in Physics, 4, 177-184, 2014.

[11] Yindoula, J.B., Massamba, A. and Bissanga, G., "Solving of Klein-Gordon by Two Methods of Numerical Analysis", Journal of Applied Mathematics and Physics, 4, 1916-1929,2016.

[12] Shahen, N.H.M., Foyjonnesa and Habibul Bashar, Md. "Exploration on traveling wave solutions to the 3rd-order klein-fock-gordon equation (KFGE) in mathematical physics", International Journal of Physical Research, 8(1), 14-21,2019.

[13] Baskonus H.M., Bulut H. "On the complex structures of Kundu-Eckhaus equation via improved Bernoulli sub-equation function method”, Waves in Randomand Complex Media. 25(4) 720-728. 2015.

[14] Düşünceli, F. "Solutions for the Drinfeld-Sokolov Equation Using an IBSEFM Method", $M S U$ Journal of Science, 6(1), 505-510,2018.

[15] Düşünceli, F. "New Exponential and Complex Traveling Wave Solutions to the KonopelchenkoDubrovsky Model”, Advances in Mathematical Physics, Article ID 7801247, 9 pages, 2019. https://doi.org/10.1155/2019/7801247. 
[16] Düşünceli, F. "New Exact Solutions for the (3 + 1) Dimensional B-type KadomtsevPetviashvili Equation”. Erzincan Üniversitesi Fen Bilimleri Enstitüsü Dergisi, 12 (1), 463468, 2019.

[17] Düşünceli, F. Çelik, E., Aşkın, M. and Bulut, H. "New Exact Solutions for the Doubly Dispersive Equation Using an Improved Bernoulli Sub-Equation Function Method", Indian Journal of Physics, 1-6, 2020. 